\title{
Topologic mixing on a microfluidic chip
}

\author{
Hao Chen and Jens-Christian Meiners ${ }^{\text {a) }}$ \\ Department of Physics and Biophysics Research Division, University of Michigan, \\ Ann Arbor, Michigan 48109-1120
}

(Received 11 November 2003; accepted 23 January 2004)

\begin{abstract}
Mixing two liquids on a microfluidic chip is notoriously hard because the small dimensions and velocities on the chip effectively prevent turbulence. We present a topological mixing scheme that exploits the laminarity of the flow to repeatedly fold the flow and exponentially increase the concentration gradients to obtain fast and efficient mixing by diffusion. It is based on helical flow channels with opposite chiralities that split, rotate, and recombine the fluid stream in a topology reminiscent of a series of Möbius bands. This geometry is realized in a simple six-stage, two-layer elastomer structure with a footprint of $400 \mu \mathrm{m} \times 300 \mu \mathrm{m}$ per stage that mixes two solutions efficiently at Reynolds numbers between 0.1 and 2 . This represents more than an order of magnitude reduction in the size of microfluidic mixers that can be manufactured in standard multilayer soft lithography techniques. (c) 2004 American Institute of Physics. [DOI: 10.1063/1.1686895]
\end{abstract}

Microfluidic devices and systems are becoming increasingly popular for applications all across the life sciences. Multilayer soft lithography has attracted particular attention because it allows not only inexpensive large-scale production of microfluidic chips from replication molds, but also the incorporation of active elements such as pumps and valves on the chip. ${ }^{1}$ With the ever shrinking dimensions of microfluidic components, a remarkably large scale of integration can be achieved. Recently, Thorsen et $a l .{ }^{2}$ demonstrated a multiplexer with 1000 sample cells that are accessed through 3574 valves on a single chip. Despite these advances toward a highly integrated lab on-a-chip, one key component has proven very difficult to miniaturize: the fluidic mixer. The principal obstacle is that at typical microfluidic dimensions ( $l=100 \mu \mathrm{m}$ feature size) and flow rates $(v=1 \mathrm{~mm} / \mathrm{s})$ the Reynolds number $\mathrm{Re}=l \rho v / \eta \approx 0.1$ for an aqueous solution (density $\rho=1 \mathrm{~g} / \mathrm{cm}^{3}$, viscosity $\eta=0.001 \mathrm{Ns} / \mathrm{m}^{2}$ ) is so low that all flow is laminar and turbulence cannot be achieved. Diffusion, on the other hand, is too slow to be effective. In order to mix two protein solutions with a diffusion coefficient $D=2 \times 10^{-11} \mathrm{~m}^{2} / \mathrm{s}$ at the flow parameters above, a channel length $l_{c}=0.5 \mathrm{v} l^{2} / D$ of $25 \mathrm{~cm}$ is required. To overcome this difficulty and achieve mixing on length scales compatible with microfluidic designs, several schemes have been proposed. Active mixers pump the fluid repeatedly around a circular path, ${ }^{3}$ whereas chaotic flow mixers stretch and fold the fluid flow laterally to obtain an exponential decrease in the size of the concentration inhomogeneities with channel length. ${ }^{4}$ Examples for such chaotic mixers are stirring in helical microchannels ${ }^{5}$ and chaotic flow in microchannels with herringbone relief structures on the bottom wall. ${ }^{4}$ The helical channels require atypically high Reynolds numbers $(\operatorname{Re}>10)$ to be effective, whereas the latter is relatively inefficient at inducing a lateral flow and still requires channel lengths on the order of centimeters for efficient mixing. To reduce the footprint of such a mixer, Therriault et al. ${ }^{6}$

\footnotetext{
a) Author to whom correspondence should be addressed; electronic mail: meiners@umich.edu
}

have introduced a three-dimensional vascular network of flow channels. Their 16-layer structure has a lateral size of $2 \mathrm{~mm} \times 2 \mathrm{~mm}$, but is due to its vertical complexity rather difficult to manufacture.

In a different approach, an array of microfabricated nozzles is used to create a layered stream to reduce the effective length scale for diffusive mixing. ${ }^{7-9}$ This flow laminating technique requires typically one microfluidic element for each boundary layer that is created. Thus, the required channel length for efficient mixing decreases only quadratically with the number of fluid-manipulating elements, and not exponentially as in the case of chaotic mixers.

In this article, we present a topological structure that exploits the laminarity of the flow to repeatedly fold the flow and double the lateral concentration gradient deterministically in a very compact geometry. Unlike a conventional laminar mixer that adds one boundary layer per microfluidic element to the stream, our topology performs a series of Baker's transformations on the concentration profile. This creates a layered fluid stream in a process that decreases the required channel length exponentially with the number of microfluidic elements on the chip. The resultant exponential decrease in the size of the inhomogeneities is the same as for the chaotic mixers; but efficient mixing can be achieved with channel lengths on the order of millimeters.

Our scheme uses a series of microfluidic elements (splitters, turns, combiners) that retain the concentration profile across the channel. We split the flow into two identical streams, and through a series of turns, rotate the concentration profile by $\pi / 2$ in opposite directions in each channel. Upon recombination, the concentration pattern and gradients are doubled. The basic topologic structure to achieve this is somewhat akin to a Möbius band: A surface vector on the band, or here, the concentration gradient vector in the flow channel, is rotated in a different direction depending on the chirality of the chosen path, even though they start at and reach the same locations. Figure 1(a) shows a realization of this topology: The two fluid streams are combined, split outof-plane, rotated in opposite directions, and recombined, 
(a)

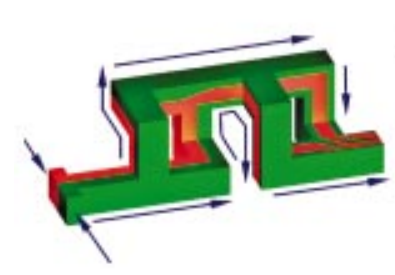

(c)

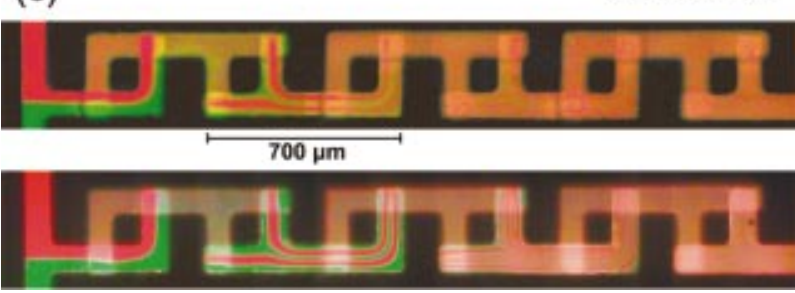

FIG. 1. (Color) (a) Topologic structure for microfluidic mixing. Two different solutions are combined in a $T$-junction. The fluid flow is repeatedly split, rotated, and recombined as indicated by the arrows. (b) Schematic crosssection of an assembled mixing chip. The two principal elastomer layers are fused together and anchored with a third elastomer layer on a glass cover slip. The chip is embedded in a block of epoxy resin for additional mechanical stability; steel tubes provide the inlets and outlet. (c) Mixing of two fluorescently labeled protein solutions in a six-stage mixer at a flow rate of $1 \mathrm{~mm} / \mathrm{s}$, corresponding to a Reynolds number of 0.1. (top). Mixing of the same dyes in an aqueous $54 \%$ glycerol solution with ten-fold higher viscosity at a flow rate of $10 \mathrm{~mm} / \mathrm{s}$, maintaining the same Reynolds number of 0.1 (bottom).

folding over the concentration profile and doubling the lateral gradient. A subsequent similar stage doubles the gradient once more and returns the fluid stream to the original plane.

As such a truly three-dimensional structure is hard to manufacture, we simplified the design by eliminating the straight out-of-plane runs. Our design uses two planar layers that are sandwiched and fused together, as shown in the cross-section Fig. 1(b). Sufficient out-of-plane rotation is obtained where the channels in the different layers overlap, as long as the cross section of the channels is sufficiently square. Our channels are $100 \mu \mathrm{m}$ wide and $70 \mu \mathrm{m}$ deep, and each stage of the mixer has a footprint of $400 \mu \mathrm{m}$ $\times 300 \mu \mathrm{m}$.

The microfluidic chips are fabricated by replication molding of a silicone elastomer (RTV 615 A and B, General Electric, Waterford, NY) from a master mold. The master molds are manufactured using a rapid-prototyping approach, ${ }^{10}$ in which a $70 \mu \mathrm{m}$ thick layer of patterned photoresist (SU-8 2050, Micro-Chem NANO ${ }^{\mathrm{TM}}$, Newton, MA) serves directly as the mold for the elastomer. The top layer was cast as a slab of $4-5 \mathrm{~mm}$ thickness from a 4:1 mixture of RTV $615 \mathrm{~A}$ and B, whereas the bottom layer was spin-cast to a thickness of $90 \mu \mathrm{m}$ from a 25:1 mixture of the two RTV compounds. After curing, both layers were sandwiched together under a stereomicroscope, and stainless steel tubing was inserted to provide inlets and outlets for the fluids. The elastomer chip was then anchored on a microscope cover glass. To insulate the chip from mechanical strain from the external mounting or tubing, which causes fluctuations in the flow rates as the channels expand or contract, the chip can be encapsulated in a block of epoxy resin ${ }^{11}$ (Tra-bond 2115, (a)
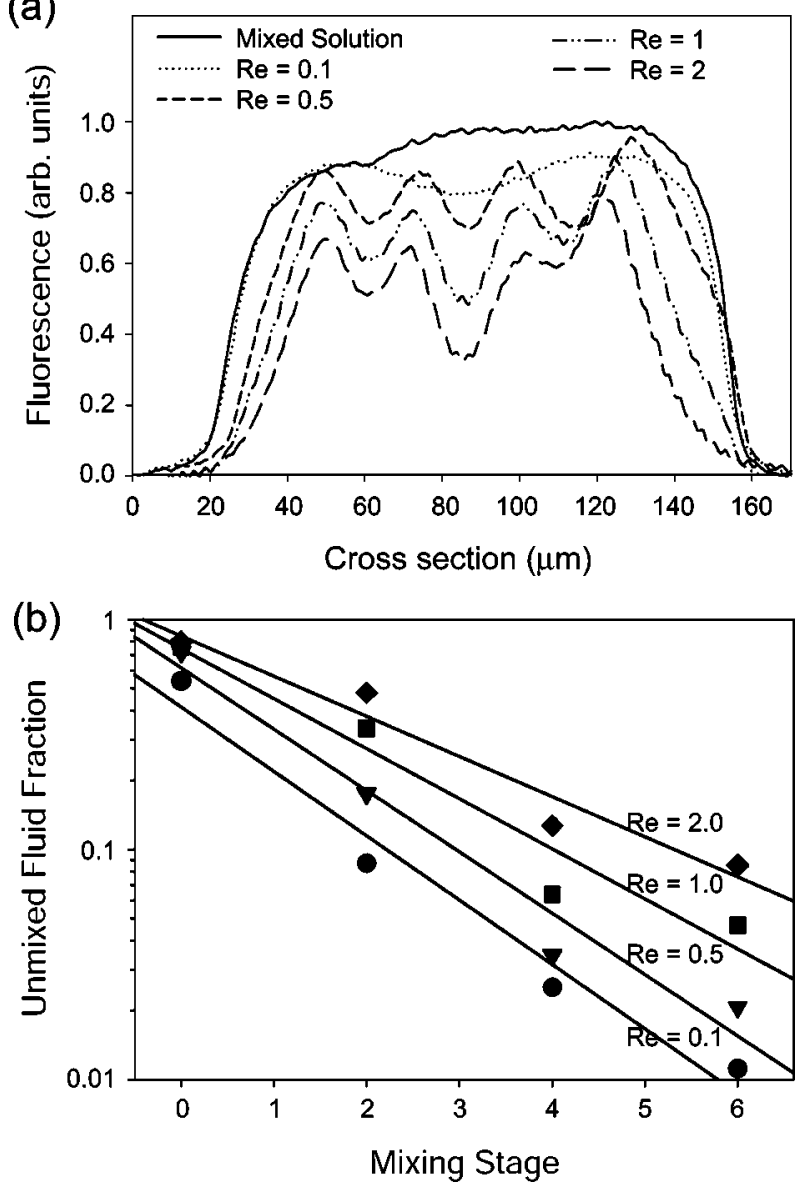

FIG. 2. (a) Fluorescence across the flow channel after two mixing stages when a $\mathrm{Ca}^{2+}$-sensitive dye is mixed with a $\mathrm{CaCl}_{2}$ solution at different flow velocities, after the background is subtracted. The fluorescence of a premixed solution is shown for comparison. (b) Fraction of unmixed fluid at each stage of the mixer for different flow rates, as determined from the fluorescence measurements. Linear regression lines in the semilogarithmic display indicate the exponential increase in mixing efficiency with channel length.

Tra-Con Inc., Bedford, MA). A cross section of the entire chip assembly is shown in Fig. 1(b).

To demonstrate mixing of two protein solutions on the chip, the chip is mounted on an inverted optical microscope. Two kinds of fluorescently labeled streptavidin (Streptavidin AlexaFluor488 and Streptavidin AlexaFluor568, Molecular Probes) were dissolved at a concentration of $1 \mathrm{mg} / \mathrm{ml}$ in PBS $\left(8 \mathrm{mM} \mathrm{Na} 2 \mathrm{PO}_{4}, 1.5 \mathrm{mM} \mathrm{KHPO}{ }_{4}, 2.7 \mathrm{mM} \mathrm{KCl}, 130 \mathrm{mM}\right.$ $\mathrm{NaCl}, \mathrm{pH} 7.3)$ and injected into the flow channels with syringe pumps at flow rates of $15 \mu \mathrm{l} / \mathrm{hr}$ each. The fluorescence was imaged onto a commercial color charge-coupled device camera using a two-color fluorescence filter set (FITC/Texas Red, Chroma Technology, Rockingham, VT).

Figure 1(c) shows the mixing of the two fluorescently labeled protein solutions in a six-stage mixer. Initially, the fluids are combined in a $T$-junction, exhibiting one sharp boundary layer. After the first two mixing stages, four interfaces, now broadened by diffusion. After three stages or a device length of $1.2 \mathrm{~mm}$, the liquids are well mixed. Under our conditions, the Reynolds number and Peclet numbers are $\mathrm{Re}=0.1$, and $\mathrm{Pe}=0.69$, respectively, which indicates that diffusion is the dominant mixing process at the interfaces. By increasing the viscosity of the solution and increasing the 
flow rate, we can slow down and decrease the efficiency of diffusion while maintaining a Reynolds number of $\operatorname{Re}=0.1$. The second micrograph in Fig. 1(c) shows the mixing of the same fluorescent dyes at ten-fold higher viscosity and flow rate. Whereas the channel length required for purely diffusive mixing in a linear channel in this scenario increases hundred-fold, our mixer obtains efficient mixing after five stages, or an approximate doubling of the device length due to the favorable exponential scaling of our topologic scheme.

To quantify the performance of our mixer, we mixed a $\mathrm{Ca}^{2+}$ sensitive dye (Fluo-4, Molecular Probes) with a $\mathrm{CaCl}_{2}$-solution on the chip and used the resultant increase in fluorescence to determine the degree to which the solutions are mixed. ${ }^{12}$ The dye and the $\mathrm{CaCl}_{2}$ were dissolved in morpholino propanesulfonate buffer $(20 \mathrm{mM}, \mathrm{pH} 7.2)$ at concentrations of 54 and $70 \mu \mathrm{M}$, respectively. Fluorescence images of the mixing fluid streams were captured at various stages of the mixer at Reynolds numbers ranging from 0.1 to 2; sample cross sections are shown in Fig. 2(a). The fluorescence background of the dye alone was determined from a premixed solution of the dye and a $\mathrm{Ca}^{2+}$-free buffer, and subtracted before the net increase in fluorescence due to mixing was determined by integration. Comparison with a $\mathrm{Ca}^{2+}$-activated reference solution, which was mixed before it was introduced into the chip, yields a measure for the remaining unmixed fluid volume at each stage of the mixer. The result, as shown in Fig. 2(b), indicates that this unmixed volume indeed decreases exponentially with the number of mixing stages, or equivalently, channel length, for the entire range of flow velocities ranging from $R e=0.1$ to $R e=2$, and $\mathrm{Pe}=0.69 \mathrm{Pe}=13.9$.
In conclusion, we have demonstrated that effective microfluidic mixing can be achieved on short lengths scales with a purely laminar flow through a flow-folding topologic structure. Favorable scaling laws ensure efficient mixing even under unfavorable conditions, such as a high molecular weight or high viscosity. While the topologic principle behind the mixer is independent of the chosen microfluidic platform technology, we have shown that an efficient device can be readily manufactured by standard planar multilayer soft lithographic techniques.

This work was supported through NIH Grant No. GM65934-01 and the Alfred P. Sloan Foundation. We thank R. Kennedy for access to his clean room facilities.

${ }^{1}$ M. A. Unger, H. P. Chou, T. Thorsen, A. Scherer, and S. R. Quake, Science 288, 113 (2000)

${ }^{2}$ T. Thorsen, S. J. Maerkel, and S. R. Quake, Science 298, 580 (2002).

${ }^{3}$ J. Liu, M. Enzelberger, and S. R. Quake, Electrophoresis 23, 1531 (2002).

${ }^{4}$ A. D. Stroock, S. K. W. Dertinger, A. Ajdari, I. Mezic, H. A. Stone, and G. M. Whitesides, Science 295, 647 (2002).

${ }^{5}$ R. H. Liu, M. A. Stremler, K. V. Sharp, M. G. Olsen, J. G. Santiago, R. J. Adrian, H. Aref, and D. J. Beebe, J. Microelectromech. Syst. 9, 190 (2000).

${ }^{6}$ D. Therriault, S. R. White, and J. A. Lewis, Nat. Mater. 2, 265 (2003).

${ }^{7}$ F. G. Besoth, A. J. deMello, and A. Manz, Anal. Commun. 36, 213 (1999).

${ }^{8}$ V. Hessel, S. Hardt, H. Lowe, and F. Schonfeld, AIChE J. 49, 566 (2003).

${ }^{9}$ S. K. W. Dertinger, D. T. Chiu, N. L. Jeon, and G. M. Whitesides, Anal. Chem. 73, 1240 (2001).

${ }^{10}$ D. C. Duffy, J. C. McDonald, J. A. Schueller, and G. M. Whitesides, Anal. Chem. 70, 4974 (1998)

${ }^{11}$ H. Chen, D. Acharya, A. Gajraj, and J. C. Meiners, Anal. Chem. 75, 5287 (2003).

${ }^{12}$ H. Song, J. D. Tice, and R. F. Ismagilov, Angew. Chem., Int. Ed. 42, 768 (2003). 